\title{
Patients' experiences of healing a venous ulcer were described as "forever healing" which affected their activities and treatment
}

Chase SK, Melloni M, Savage A. A forever healing: the lived experience of venous ulcer disease.J Vasc Nurs 1997 Jun;15:73-8.

\section{Question}

How do patients experience life with a venous leg ulcer (a leg ulcer caused by chronic venous insufficiency)?

\section{Design}

Phenomenology.

\section{Setting}

An ambulatory surgical clinic in an urban teaching hospital.

\section{Patients}

37 of 54 patients, whose venous ulcer dressings were changed weekly by clinic nurses, agreed to participate in the study. Patients were usually treated using an Unna's boot.

\section{Methods}

Data were collected for 1 year and included weekly measurements of wound size using acetate tracings; activity and pain logs kept by 12 patients; medical chart reviews for concomitant conditions, previous ulcers, and related emergency department and hospital admissions; and weekly participant observation sessions with patients to follow the course of healing over time. 7 patients were interviewed using open ended questions about their experience of venous ulcers. Interviews and field notes were analysed for themes using van Manen's approach to phenomenological analysis. Themes were then collapsed into categories. Consistency and confirmability of findings were tested by the study authors who reread the data and tested its fit with the final themes. The interview data were triangulated with the observation data and the findings were tested with study participants. Patients and staff were presented with the major categories and asked for responses to validate the conclusions.

\section{Main results}

For the 54 patients who had weekly dressing changes there were 38 venous ulcers that healed over a mean time of 31 weeks. 4 major themes emerged from the analysis of the 37 patients' experiences of healing venous ulcers. The first theme, a forever healing process, included the experiences of a lengthy healing process, ulcer recurrences, and chronic pain. The second theme, limits and accommodations, referred to the loss of mobility, which resulted in the loss of work for several patients; the limitations imposed by treatment with an Unna's boot; the changes in body image related to having an open sore; and the fear of amputation for patients whose ulcers did not heal and who did not understand the underlying condition. The third theme, powerlessness, included feeling resigned to the slow healing of the wound and the inevitability of recurrence, feeling uncertain that the wound would ever heal, and not understanding the causes of the ulcer nor how the treatment helped. The fourth theme, who cares?, related the various ways patients assumed responsibility for managing their venous ulcers. Some patients did not feel a sense of ownership of their condition or of their treatment plan, which led to delays in seeking help and to patients being less vigilant in preventing or noting venous ulcer recurrences.

\section{Conclusions}

Venous leg ulcers were perceived as forever healing by patients who also experienced restricted mobility, interference with employment and hobbies, altered body image, and pain. A sense of powerlessness was associated with the slow healing and high recurrence rates of venous leg ulcers. Patients varied in their abilities to participate in managing their leg ulcer.

Source of funding: Office of Research Administration, Boston College.

For article reprint: Dr $S$ K Chase, School of Nursing, Boston College, 140 Commonwealth Avenue, Chestnut Hill, MA 02167-3812, USA. Fax +1 6175520745.

\section{Commentary}

The study by Chase $e t$ al is a welcome addition to the literature on leg ulcers. It adds depth to several European studies which measured quality of life quantitatively. ${ }^{1-3}$ It also enhances the work by Lindholm which identified pain as the "worst thing" about the ulcer. ${ }^{4}$

The authors have described their methodology and the means by which they validated their study. This is helpful because many readers may not be familiar with this type of rigorous approach. Study strengths included multiple sources of data and validation of major categories by patients and staff.

Chase et al describe the profound effects that leg ulcers can have upon the lives of patients. The study encourages nurses to reflect upon their own patients and to consider how they might more effectively identify patient needs. In particular, it highlights how uncertainty governs the lives of many patients with leg ulcers. The study also shows the effect of chronic pain and reduced mobility on these patients. It would have been interesting to know the age range of patients, particularly in the context of participation in treatment and mobility problems, given that there is usually some loss of mobility in the elderly.

Further research is needed to identify strategies to effectively address the issues raised in the study such as uncertainty, powerlessness, limitations, and involvement.
Carol Dealey, RGN, RCNT, BScN University Hospital Birmingham NHS Trust Birmingham, UK

1 Cullum N, Roe B, Hamer C. The effect of leg ulceration on quality of life in the elderly. In: Harding KG, Dealey C, Cherry G, et al, editors. Proceedings of the 3rd European conference on advances in wound management. London: Macmillan Magazines, 1994:57.

2 Lindholm C, Bjellerup M, Christensen O, et al. Quality of life in chronic leg ulcer patients. An health profile. In: Harding KG, Dealey $\mathrm{C}$, health profile. In: Harding KG, Dealey C, Cherry G, et al, eds. Proceedings of the 3rd European Conference on advances in wound manageHofman D, Ryan TJ, Arnold F, et al. Pain in Hofman D, Ryan TJ, Arnold F, et al. Pain in
venous ulcers.J Wound Care 1997;6:222-4.

4 Lindholm C. Pain in patients with leg ulcers. In: Leaper D, Cherry G, Cockbill S, et al. Proceedings of the European Wound Management Association Spring Meeting. London: Macmillan Magazines, 1998:101-2. 